\title{
On timing events in computer-controlled experiments
}

\author{
JOSEPH MARKOWITZ AND RAYMOND S. NICKERSON, \\ BOLT, BERANEK \& NEWMAN, INC., 50 Moulton Street, \\ Cambridge, Mass. 02138
}

This paper considers the problem of timing events in computer-controlled experiments. A digital clock is defined as a time-mark generator or "ticker" and a counter. A discussion of time-mark generators commonly used in computers is followed by a consideration of several ways in which computer clocks are implemented and used. A framework for the latter discussion is provided by a classification of clocks in terms of whether the ticker or the counter (either or both) is internal or external to the computer.

With the growing availability of computers, psychologists have sought and found ways to use them advantageously in research. Early applications in such areas as data analysis, stimulus generation, process simulation and automated instruction have been reviewed fairly extensively (Borko, 1962; Coulson, 1962; Green, 1962; Fliege, 1966). This paper is concerned with the use of computers to control the data-collection process in psychological experiments. This application appears to be gaining adherents at an impressive rate. Reports of experiments conducted under computer control are beginning to appear fairly regularly in the psychological literature. What the eventual impact of this type of computer usage will be on experimental psychology only time will tell. Our feeling is that the effect is likely to be profound. It will result, we expect, in the development of more efficient data-collection techniques and in the evolution of new principles of experimental design. More importantly, it will affect the types of problems to which experimenters will address themselves, and the nature of the theories that will result.

The particular aspect of computer-controlled experimentation with which we are concerned is that of timing. In most psychological experiments the $\mathrm{E}$ wishes to control some of the temporal properties of the situation, such as the duration of stimulus presentation. In many cases he may also want to measure the duration of events whose timing is not under his control, for example, response latencies. When a computer is used to control data collection, time may be measured in a variety of ways. We will discuss some of the techniques available and attempt to indicate their relative strengths and weaknesses.

The word clock is used in the generic sense to connote any device that can serve to measure time. The basic elements of a digital clock are a time mark generator, or "ticker," and a counter. The operation of our clock is schematized in Fig. 1. If the inter-tick interval is of duration $\Delta t$, the elapsed time since initialization is simply the value of the counter multiplied by $\Delta t$.

\section{TIME MARK GENERATION}

Three different techniques have been widely employed to generate time marks for use in computer systems.

\section{Crystal Oscillators}

An electrically excited piezoelectric crystal can be used to generate a train of pulses at a rate corresponding to the crystal's natural frequency. Resonant frequencies of crystals range up-

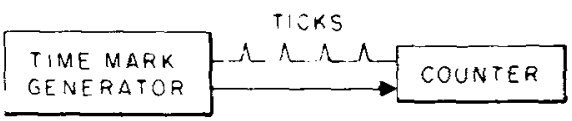

Fig. 1. A clock.

wards from a few $\mathrm{KHz}$ and depend on such factors as the size and shape of the crystal.

Crystal oscillators are commonly utilized to provide the timing pulses that are used to synchronize the basic computer operations. They have the advantage of being extremely accurate and stable. Crystals are also used as time mark generators for the "crystal clocks" that are available as optional peripheral equipment with many computer systems. As the time mark generator for a general purpose clock, a crystal has the limitations of an inflexible time base; that is, its tick rate cannot be modified except by replacing the crystal with another having a different natural frequency.

\section{Line Current}

Another method of time mark generation makes use of alternating line current. The common $60 \mathrm{~Hz}$ current can be used to produce a pulse every $16.67 \mathrm{msec}$; the less common $50 \mathrm{~Hz}$ current could generate a pulse every $20 \mathrm{msec}$. Although this is far too slow a time mark generator to be used to synchronize the basic computer operations, it is a perfectly good ticker for timing events when accuracy to within about $20 \mathrm{msec}$ is acceptable. For many psychological applications this is more than adequate; for others it may be somewhat less precise than is desirable.

This method also has the disadvantage of an inflexible time base or tick rate. A minor inconvenience is the awkward (16.67 msec) time quantum that is obtained with a $60 \mathrm{~Hz}$ current. A trivial point perhaps, but nevertheless it is a nuisance factor which does not add to the attractiveness of the scheme.

\section{Astable Multivibrators}

A third method for generating time marks involves the use of an electronic circuit known as an astable multivibrator. Because of the flexibility of this method we shall describe it in some detail.

The basic building block is the logical NAND-gate. Figure 2 shows a two-input NAND-gate, completely described by its truth table, and the preference of the inputs to assume the value 1 when nothing is connected to them.

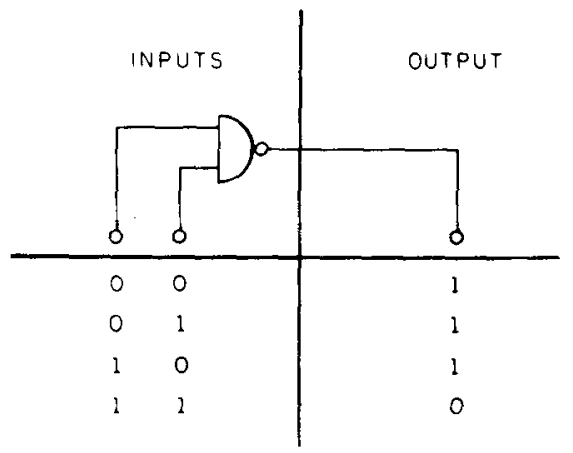

Fig. 2. A two-input NAND gate and its truth table. 
We could generalize to an n-input NAND-gate and also handle the cases where some of the inputs are unused. Instead, consider the case of a single input NAND-gate (a two-input gate with one input unused). Such a one-input gate provides the logical function of negation, and is often called an inverter.

If we take two one-input NAND-gates and cross-couple them-connect the output of each to the input of the other as shown in Fig. 3-the configuration is called a multivibrator. This device can be in one of two states, a state being defined by the values at the nodes. One state is shown in parentheses, the other not.

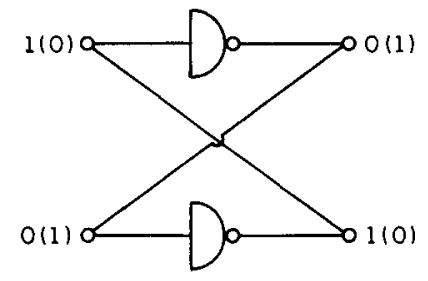

Fig. 3. Two cross-coupled NAND gates-a multivibrator. See text.

If both coupling paths are permanent, the device is stable in whichever state we find it. If we forcibly change one of the inputs momentarily, the device changes to the other state and remains that way until disturbed again. Because it is stable in either state, it is termed a bistable multivibrator, "flip-flop," or simply a "flop."

Suppose that one coupling were not permanent but instead existed for a time $\Delta t$ after a momentary change at one of the inputs. Then the device would be stable only in one of its states (recall that an input left disconnected, or decoupled has a preference for the value 1). Such a device with one permanent coupling, but with one transitory coupling, is called a monostable multivibrator or "one-shot." It will remain in the unpreferred state only $\Delta t \mathrm{sec}$ after it has been momentarily forced there.

Finally, suppose that both coupling paths are transitory, one existing for duration $\Delta t_{1}$, and the other for duration $\Delta t_{2}$, after a perturbation. This device, is stable in neither state, and regularly changes back and forth between them. It is known as an astable multivibrator or "oscillator," generating a periodic waveform of wavelength $\Delta t_{1}+\Delta t_{2}$, with duty cycle

$$
\Delta t_{1} /\left(\Delta t_{1}+\Delta t_{2}\right)
$$

To use an astable multivibrator as a clock ticker, we would adjust the parameter $\Delta \mathrm{t}_{1}$ to provide an acceptable pulse width as required by the computer and/or counter logic. The parameter $\Delta t_{2}$ would then be adjusted so that the period $\Delta t_{1}+\Delta t_{2}$ satisfies the needs of the experiment, providing our basic timing interval or time base. For example, if we wished to time events to millisecond accuracy, we would set the period equal to $1.0 \mathrm{msec}$. Unfortunately, the term millisecond accuracy is somewhat ambiguous. If we mean "to the nearest millisecond," then a 1.0 msec time base suffices, inasmuch as realizable accuracy can be \pm $0.2 \%$. If our demands are more stringent, three approaches suggest themselves, two of which are really equivalent, as we shall see.

One thing we could do is use a crystal controlled "clock" (sic) which will give 20 times the accuracy at twice the cost. The disadvantage is a loss in flexibility of the time base. Changing the time base means changing the crystal which really means acquiring a new crystal-controlled "clock."
An alternative is to use the ticks of an astable multivibrator to complement a flip-flop-that is, each tick (or transition from logical 1 to logical 0 ) causes the flip-flop to change state. Now if we look at an output of that flip-flop we note that it produces a tick on every other tick of our astable multivibrator. What we have done is to double the period, or halve the frequency. By complementing a second flip-flop with the ticks of the first we again double the period or, equivalently, halve the frequency again. This technique is extendable and called frequency division. The divisor is $2^{n}$, where $n$ equals the number of successive flip-flop stages. With respect to accuracy, the process of frequency division can be viewed as taking $2^{\text {n }}$ times as many observations, with a standard deviation proportional to the period. Thus, more and smaller inter-tick intervals might reduce the variability by as much as $2^{-n / 2}$. This assumes that the auto-correlation of the error approaches zero-that the variability is of high frequency - which is generally not the case. There is also the assumption that the flip-flop stages are perfect. In fact, they may incur propagation delays and associated variability. Typical propagation delays are on the order of 50 nanosec $\pm 10 \%$. The nth flip-flop, driven by $n-1$ preceding stages, suffers a delay approximately equal to (n-1) 50 nanosec $\pm 10 \%$. There are techniques for avoiding a cumulative delay, but we shall not discuss them.

The third thing that might occur is to time things more accurately than need be, and round off later. This is almost precisely what was done above. To implement frequency division, we built a counter and threw away the least significant digits. Instead of referring to the technique as frequency division, we might have said that we "counted down" by $2^{n}$.

Let us see in what sense the implementation of frequency division involved building a counter. Figure 4 shows a series of ticks feeding into successive complementing flip-flop stages. Each

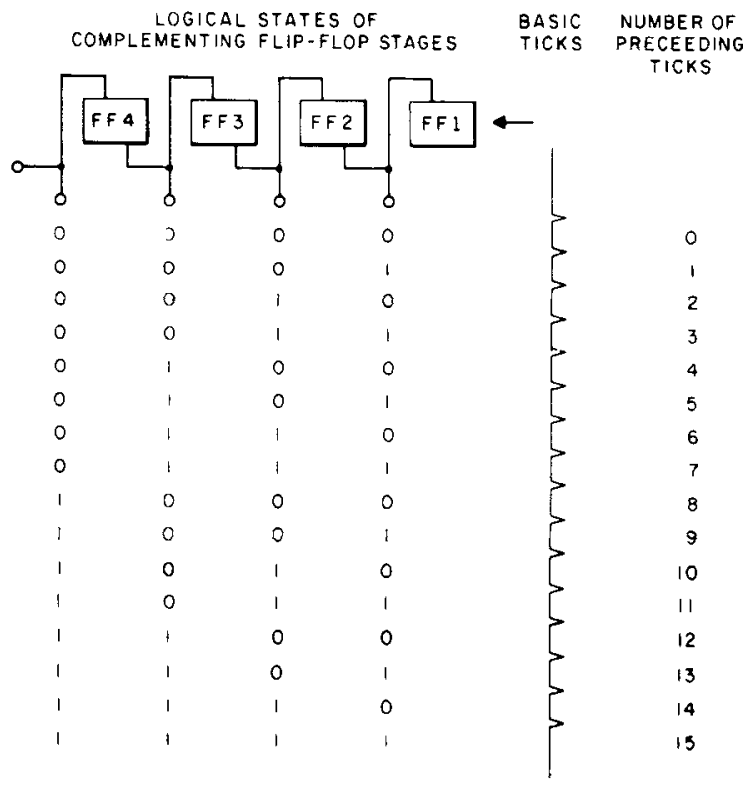

Fig. 4. Implementing frequency division with complementary flip-flops - a counter.

flip-flop changes state when its right hand neighbor makes the transition from a logical 1 to a logical 0 . The states of each flip-flop are tabulated for each succeeding tick, and the ticks are 
numbered. For the reader's convenience we have started numbering the ticks at zero, and assume that the initial states of all flops were logical $O$. If the states of the flops are considered as a number word, it is the binary equivalent of the tick number.

\section{CLASSIFICATION OF CLOCKS}

There are various ways in which clocks might be classified. One scheme follows from the fact that the ticker or the counter (either or both) may be internal or external to the computer.

\section{Internal Ticker-Internal Counter}

Virtually all computers are equipped with some type of time mark generator (usually a crystal oscillator), which is used to control the timing of the events involved in the execution of instructions. It is natural to consider the possibility of using this as the ticker for a clock with which to time events of interest to E. There are two ways in which the computer's basic timing circuitry might be used.

The first method does not involve the timing pulses directly, but assumes their existence. For most computers the events involved in the execution of any instruction occur in synchrony with pulses generated by the timing circuitry; the time required to execute any particular instruction is precisely fixed and known. One can then determine the time required to execute the individual instructions (taking due account of program loops). To measure or control the duration of events of interest, one must set up one or more counters in memory and include within his program instructions for initializing, incrementing and reading them under the appropriate conditions. Conceptually this is a simple solution to the time-keeping problem; in practice it places an onerous burden on the programmer and is not recommended.

\section{Internal Ticker-External Counter}

A second and more direct use of the computer's basic timing circuitry would involve reading the timing pulses of the internal time mark generator into a counter of our own manufacture. This might appear to be a straightforward scheme, but it is a poor one for several reasons. If we tap the computer's own time mark generator we run the risk of causing malfunctions in the computer itself. Because of the primal nature of the time mark generator to all of the computer's operations such malfunctions may show up in strange ways. They may be intermittent, random, or instruction-dependent-in general, difficult to detect and trouble-shoot, and interactive with warranties and service contracts.

Assuming we could safely tap the computer's time generator directly, it would almost always be a futile exercise. Putting the computer's time base into an external counter of our own gives us inherently unuseable timing accuracy. There is no way the computer could service such a clock between counts if the counts come along as fast as the computer's instructions. Indeed, because the counter is external, the computer must service it by input-output transfers, which are invariably slower than the basic computer cycle time. To get around this problem we might interpose a shift register or another counter between the ticker and the counter of our clock so that the clock counter would be incremented only every nth timing pulse. This, however, is an inefficient stratagem; there are preferred solutions to the problem.

Before discussing the next two types of clocks it will be necessary to digress long enough to introduce the notion of an interrupt system. Most modern computers are equipped with an interrupt capability as an option if not as a standard feature. Such a system provides a means of interrupting a running program long enough to allow the computer to service some external device. Whenever an interrupt occurs, several things happen, two of which are relevant to this discussion. (1) The contents of certain registers of the control and arithmetic units (accumulator, program counter, etc.) are stored in specific memory registers reserved for that purpose. (2) Control is transferred to a "service" routine-a program or program segment that specifies what is to be down in response to the interrupt. When the service routine is completed, the original contents of the control and arithmetic registers can be restored, and control transferred back to the instruction that was about to be executed when the interrupt occurred, and the program continues.

One basic distinction is that between interrupt systems which have only a single channel, and those which have several. In the latter case, the channels usually are assigned priorities. If one device requests an interrupt while another is being serviced, whether the second request will be granted immediately or only after the servicing of the first is completed, will depend on the relative priorities of the two channels involved. The availability of a priority internupt system simplifies the clock problem considerably.

\section{External Ticker-Internal Counter}

Assuming a multi-channel priority interrupt capability, there are several ways in which a clock might be implemented. One approach would be to use an external ticker and an internal programmed counter. Thus, a crystal oscillator or multivibrator might be connected to one of the interrupt channels. (The priority of the channel with which the ticker is associated will depend, among other things, on the importance assigned to accuracy in our clock. Undoubtedly, the priority of the clock channel will be relatively high.) Assume for the sake of illustration that our ticker is generating a pulse once every millisecond, and is connected to the highest priority channel in the system. Thus, whenever that channel is activated (channels usually are activated and deactivated under program control), the running program will be interrupted every millisecond. Each time that an interrupt occurs the service routine will simply increment the contents of a memory register, which is holding the count, and return control to the main program.

Precisely how we might use such a clock would depend on what it is that we wish to time. If for example we wished to control the duration of a stimulus presentation, we might set the count to zero, activate the channel to which the ticker is connected, and enter the program loop that controls the presentation of the stimulus. The stimulus presentation would then be terminated as soon as the clock count reached a prespecified value.

The test for completion could be made in either of two places. (1) in the clock service routine or (2) in the stimulus presentation loop. In the former case instructions might be included within the counter routine that would compare the size of the count with the prespecified criterion value and set the return pointer on the basis of the outcome of that comparison. Thus, as long as the count remained smaller than the criterion the counter routine would return control to the instruction in the stimulus presentation loop that was to be executed when the interrupt occurred. As soon as the count equalled the criterion, the counter routine 
would transfer control to the appropriate place in the main program for terminating the stimulus presentation. If, on the other hand, the test of count size were made within the stimulus presentation loop, the counter routine would always return control to the point at which the internuption occurred.

The first alternative will, in general, give greater timing accuracy because the stimulus will be terminated as soon as the criterion count is reached. (If the test is incorporated in a relatively large stimulus presentation loop, the clock counter might be incremented several times between successive tests.) However, this alternative also increases somewhat the overhead of time-keeping, since it means adding instructions to the clock service routine. A major advantage in performing the test within the stimulus presentation loop is the greater generality thereby given to the clock service routine; the latter need not then be modified for each particular case.

The problem of determining the duration of an event whose timing is not under computer control requires a slightly different procedure. Suppose for example that we wished to measure a response latency. In this case the S's response mechanism might be connected to one of the interrupt channels (other than the clock's channel). A signal on this channel would indicate that the response had been made and that the clock should be read. What needs to be done at this point depends on exactly how the clock is being used. If the clock count had been set to zero just before the presentation of the stimulus (as it probably would have been if it were being used only to measure the response latency) then it would be necessary simply to note the count when the interrupt from the response mechanism occurred. It frequently happens however that $\mathrm{E}$ will want to time several overlapping events with the same clock. In this case he would not necessarily set the clock count to zero at the beginning of each event. Rather he might let the clock run continuously, read the count at the beginning of an event of interest, read it again at the completion of that event and compute the event duration by taking the difference between the two readings.

So far we have been discussing a clock implementation scheme in which the ticker is a piece of hardware outside the computer and the counter is a little program. Incrementing the clock count in this case is a program function. One of the disadvantages of this method is that counting ticks takes time. Thus, if the time required to execute the counter program is a large enough fraction of the time between ticks, $\Delta t$, the computer could be spending most of its time just operating the clock. (Obviously, if the execution time of the counter program were greater than $\Delta t$, the scheme would not work at all.) For most psychological applications this will not be a problem, however. Assuming (1) that a $1 \mathrm{KHz}$ ticker will give sufficiently precise measurement for most experiments, (2) that the computer's cycle time is not greater than $5 \mu \mathrm{sec}$, and (3) that the counter program is no more than 102 -cycle instructions (which is a generous allowance) this method of measuring time would require $1 / 10 \Delta t$ to run the clock at worst. In most modern machines, it will in fact be possible to do much better than that, both because the cycle time is considerably less than $5 \mu \mathrm{sec}$ and because fewer than 10 instructions are necessary in the counter program.

A slightly different method to implement clocks of the external ticker-internal counter type is the following. Associated with each clock are one or more memory registers that are reserved for use as counters. That is, each ticker has a direct portal to one or more specific memory locations. These registers are addressable as are memory registers in general, hence their contents may be read or modified by program. Typically, this type of clock can be turned on or off ("armed" or "disarmed," "activated" or "deactivated," "enabled" or "disabled.") This operation is also controlled by program. Whenever the clock is on, however, it is automatically counting, the count rate being determined, of course, by the frequency of the ticker.

Usually this type of clock has an alarm feature that works in one or both of the following ways. (1) An interrupt is generated internally when a count becomes zero. Let us assume for the sake of illustration that the ticker ticks every millisecond. Thus, if the user wishes to produce a stimulus of $500 \mathrm{msec}$ duration he would set the counter to -500 ( 500 if the counter counts down instead of up), turn on the clock, and initiate the presentation of the stimulus. The interrupt request from the clock channel would then be the signal to terminate the stimulus presentation. (2) An interrupt is requested when the content of a counter register equals the content of an "alarm" register. The latter is a special memory register reserved for this alarm function. If in this case the user wished to produce a stimulus of $500 \mathrm{msec}$ duration he might do so by first reading the value of the counter register, adding 500 to that value and depositing the sum in the alarm register just before presenting the stimulus. He would then terminate the stimulus presentation as soon as an interrupt was requested indicating that the content of the counter register equalled that of the alarm register.

\section{External Ticker-External Counter}

It is possible, and often preferable, to have the entire clock-ticker and counter-outside the computer. To construct such a clock one might simply feed the output of a crystal oscillator or a multivibrator into a binary counter. Such an arrangement has the obvious advantage that it does not use up central processor time just to run the clock. The scheme does necessitate the inclusion of one or more special input-output instructions in the machine's command set. That is, one must be able to set and/or read the clock by program. Although the inclusion of both a set and a read command is not essential for most applications, it does make for programming convenience.

Typical of clocks completely external to a computer with an interrupt capability is one whose general configuration is shown in Fig. 5 below. This clock was designed for a PDP-8 computerbased psychophysical laboratory.

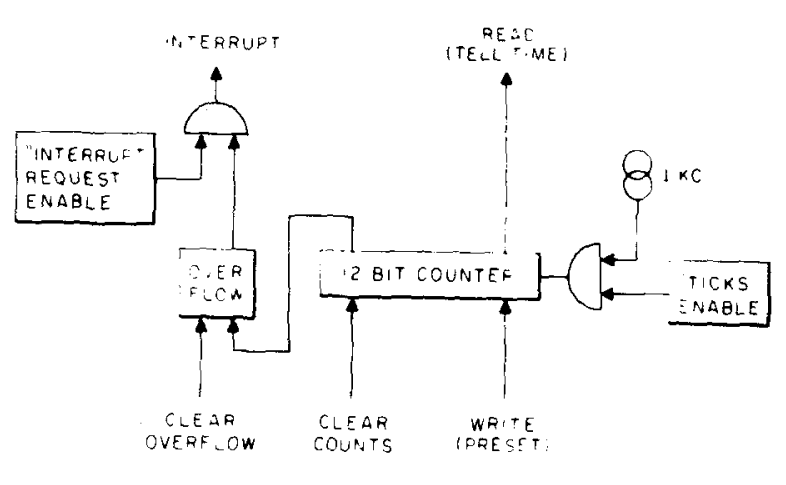

Fig. 5. Diagram of a clock designed for use in a computer-based (PDP-8) psychophysical laboratory. 
The counter is 12 bits (to match the computer word size) plus overflow, and is driven by a $1 \mathrm{KHz}$ ticker. The clock can function in either an interrogation mode (the computer reads the time-word), or in an interruption mode (the clock triggers an interrupt request when a preset time has elapsed). To facilitate the latter mode, any initial value can be written into the counter. The interrupt is generated on overflow, contingent on the status of "intermpt request enable," which is under program control. After an overflow condition is established, the counter can continue to count. The status of the overflow bit can be checked by the program (so that we can effectively have a 13-bit counter if desired) and separately cleared by the program.

The clock may be stopped and started dependent upon the status of "ticks enable." Thus, we can accumulate lapsed time for a process that starts and stops. This same logic could be used to steer one or another time-mark generator to the counter, thus providing programmed selection of time bases. Moreover, the logic is designed to interrupt, momentarily and automatically, the counter from counting while the computer is effecting a transfer to or from the counter. Things are so arranged that reading on the fly can be accomplished without missing a count.

Much of our previous discussion pointed up the merits of a computer with a multi-channel priority-interrupt capability. This clock is used on a PDP-8 which has but a single interrupt line. The technique used to bypass the single channel constraint is worth noting.

Because our S's response buttons are also tied into the same interrupt line, we have no way of knowing whether an interrupt was caused by the clock or the subject(s). We could set up status flops which could be checked by the program and would serve to answer the question. Instead, a "block data transfer" is executed on every interrupt-we read in the clock counter and all Ss' response devices. Sorting out who or what caused the interrupt is done afterwards, thus preserving timing accuracy. This technique can be extended to other external data sources; the only trick is to pack the information into as few computer words as possible so as to make the block as small as possible.

\section{CONCLUDNG REMARKS}

It seems safe to say that the clock with both ticker and counter external to the computer is to be preferred to most of the alternative possibilities considered. A possible exception is the case in which the ticker is external but has a direct portal to the memory location that is serving as the counter. The latter arrangement has the advantage that it is not necessary to effect input-output transfers when setting, reading, or manipulating counter values. Otherwise these two schemes are quite comparable.

The relative acceptability of any particular clock implementation will depend somewhat, of course, on the specifics of the system in which it is to be used. Consider again, for example, the case of an external ticker and an intemal programmed counter. This is the scheme in which each tick of the clock causes the ongoing program to be internupted long enough to execute a service program which simply increments the clock count. This method works very well provided the system has a multi-channel interrupt system so that one of the channels can be devoted exclusively to the ticker. If, however, the interrupt system has only a single channel, this clock implementation is less attractive.

The clock shown in Fig. 5 could be made somewhat more versatile with the addition of one or more addressable alarm registers, which would function in the following way. The contents of each alarm register would be compared with the clock count following each tick. Whenever a match occurred between any one of the alarm registers and the value of the clock counter, an interrupt would be requested. The interrupt request from each alarm register should be gated so that the user could enable or disable each alarm at will. Whether the added versatility and programming ease is worth the additional cost of such features depends on the uses to which a particular system is to be put and on the tradeoffs that the system's users are prepared to make. We have found the clock shown in Fig. 5 to be both adequate and convenient for the computer-controlled experiments undertaken at this facility to date.

\footnotetext{
REFERENCES

BORKO, H. (Ed.), Computer applications in the behavioral sciences. Englewood Cliff': Prentice-Hall, 1962.

COULSON, J. E. (Ed.), Prozrammed learning and computer based instruction. New York: Wiley, 1962.

FLIEGE, S. Digital coinputers In J. B. Sidowskd (Ed.), Experimental methods and instrumentation in psychology. New York: MoGraw-Him, 1966.

GREEN, B. F. Jr. Digital computers in research. New York: MoGraw-Hill, 1963.
}

NOTE

1. This work was apported by the Human Performance Branch of the NASA-Ames Rewearch Center under Contract No. NAS2-2676.

\section{An on-line computer in a visual perception laboratory'}

RALPH NORMAN HABER, DEPARTMENT OF PSYCHOLOGY, UNIVERSITY OF ROCHESTER, Rochester, N.Y. 14627

The use of a small data acquisition computer (PDP-8) is described for on-line operations in virual penception experiments. A typical program to control an experiment on short-term visual memory is presented, and the interface to connect the computer to the external stimulating and response devices is described in some detail. A brief description is given of some special purpose interface components and how the computer could be used for experimental situations other than those in visual perception.
The last 10 to 15 years have shown a rapid growth in the application of electronic components in experimental psychology laboratories. The timers, counters, controlling systems, and measuring devices now in use, although greatly improved in this past decade, are mainly extensions and outgrowths of those designed earlier. However, dramatic changes have occurred since the early 1960 's, with the advent of inexpensive digital computers that can be used on-line in an experimental laboratory. This has been a departure from the more customary use of the computers as bulk data processors and analyzers, since this new function is to run experiments which acquire data, and in the process control 